\title{
A Pitfall in Mouse Norovirus (MNV) Detection in Fecal Samples Using RT-PCR, and Construction of New MNV-Specific Primers
}

\author{
Masaru TAJIMA $^{1)}$, Yuko KOTANI ${ }^{1)}$, Tsutomu KUROSAWA ${ }^{1)}$, and Masayuki MIYASAKA ${ }^{1,2)}$ \\ 1) The Institute of Experimental Animal Sciences, Faculty of Medicine, Osaka University, 2-2 Yamadaoka, Suita, \\ Osaka 565-0871, Japan \\ ${ }^{2)}$ Laboratory of Immunodynamics, Department of Microbiology and Immunology, Osaka University Graduate \\ School of Medicine, 2-2 Yamadaoka, Suita, Osaka 565-0871, Japan
}

\begin{abstract}
The murine norovirus (MNV), which belongs to the Caliciviridae family, is prevalent in laboratory mice. Since this virus affects macrophages and dendritic cells, infected mice are not suitable for immunological investigations, making it important to detect MNV infections accurately. When we tested RNA extracts derived from mouse feces for MNV detection using nested RT-PCR with a set of MNV-specific primers reported by Goto et al. (Exp. Anim. 58: 135-140, 2009), we found that these primers amplified not only an MNV-specific signal but also amplified a relatively weak signal with a size almost identical to that of the specific signal. Analysis of the nucleotide sequence of this amplified signal revealed that it was at least $98 \%$ identical to the exophosphatase gene of a commensal bacterium, Bacteroides vulgatus. Subsequent analysis showed that the signal amplified with a pair of nested primers was from DNA derived from B. vulgatus, which is sometimes present in SPF laboratory mouse feces, and the nested primers used were both partly homologous with the $B$. vulgatus nucleotide sequence. We thus designed a new set of nested RT-PCR primers that was not cross-reactive with the $B$. vulgatus genome. PCR products amplified by the newly designed primers were at least $89.3 \%$ identical to the MNV RNA polymerase gene in all cases. Our findings demonstrated that the primer set we designed was suitable for detecting an MNV-specific signal without crossreacting with $B$. vulgatus DNA in mouse feces.
\end{abstract}

Key words: Bacteroides vulgatus, MNV-specific primer, murine norovirus, refinement, RT-PCR

\section{Introduction}

The murine norovirus (MNV) is a single-stranded positive-sense RNA virus belonging to the Caliciviridae family. This virus is highly prevalent among laboratory mice, and it is symptomless in immunologically normal mice $[3,9,10,14,17,18,23]$. Karst et al. showed that susceptibility to this virus is not substantially increased by deleting the recombination activating gene 1 (Rag1) or Rag2 gene-dependent specific immunity [7]. Geneti- cally disrupting the interferon (IFN) receptor gene or the signal transducer and activator of transcription 1 (Stat1) gene encoding a crucial signaling factor downstream of the IFN $\alpha \beta \gamma$ receptor induces lethal infection $[7,19]$, indicating that innate immunity is required for protection against this virus. Lymph node abnormalities have recently been reported in diabetes model mice infected with MNV [16]. Thus, MNV may cause various pathologic changes in disease model mice and immunodeficient mice [12]. Since MNV infects macrophages [7]

(Received 29 January 2012 / Accepted 22 November 2012)

Address corresponding: M. Tajima, The Institute of Experimental Animal Sciences, Faculty of Medicine, Osaka University, 2-2 Yamadaoka, Suita, Osaka 565-0871, Japan

(C)2013 Japanese Association for Laboratory Animal Science 
and dendritic cells [21], it is necessary to use MNV-free mice when analyzing the immune system and in experiments using immunodeficient mice.

Serological $[5,6,9,18]$ and RT-PCR assays $[2,5,6$, $10,14]$ have been widely used to detect MNV infections. In particular, the use of RT-PCR has shown that mice experimentally inoculated with an MNV strain (MNV-2, MNV-3, or MNV-4) have detectable viruses in the feces from day 2 to week 8 post infection [5], revealing that mice can become persistently infected and shed virus in the feces for prolonged periods. Recently, a real-time RT-PCR assay has been used to detect MNV RNA [1, 4, $11]$; this method has many advantages over conventional RT-PCR, including rapid assay time and the possibility of quantitative measurements, but it is not always highly sensitive [11].

In a nested RT-PCR, efficient virus detection requires test material containing a relatively high amount of virus genome that can be readily collected. While the MNV genome can be detected in various tissues, including the small intestine, cecum, large intestine, liver, mesenteric lymph nodes, spleen, and lungs [2, 6, 8, 12, 15], feces are commonly used for MNV testing because they can be collected noninvasively $[10,14,17]$. Nested RT-PCR thus requires a sensitive primer set and no nonspecific reactions. Taking advantage of the relatively small difference in the RNA polymerase gene from various MNV strains [6], Goto et al. successfully detected MNV by using a nested RT-PCR with primers targeting this region [2].

In the present study, we found that the primer set used by Goto et al. [2] to detect MNV yielded both MNVspecific and nonspecific PCR products. The nucleotide sequence of the latter was almost identical to a particular sequence derived from Bacteroides vulgatus DNA. Subsequent analysis showed that the nested primers used indeed cross-reacted with B. vulgatus-derived DNA, which is commonly found in the SPF laboratory mouse intestinal microbiota [13]. We therefore designed a new set of primers and found that nested RT-PCR using these primers provided high sensitivity in detecting MNV without cross-reacting with $B$. vulgatus. Our findings also indicated that attention should be given to incidental primer cross-reactivity with commensal bacterial DNA in RT-PCR-based MNV detection using fecal samples.

\section{Materials and Methods}

\section{Animals}

The study was conducted using 145 sentinel mice from our animal facilities. Female ICR (Crlj:CD-1) mice at 6-7 weeks old were purchased from Charles River Laboratories Japan (CRJ) (Yokohama, Japan) for use as sentinels. The microbiological data proposed by CRJ showed the mice to be free of antibodies against MNV, bacterial pathogens, and parasites. These sentinels were housed in open standard cages (S-cages, TPX, $143 \times 293$ $\times 148 \mathrm{~mm}$, Charles River Laboratories Japan) using paper bedding (ALPHA-dri, Shepherd Specialty Papers, Watertown, TN, USA). The cages with bedding were autoclaved before use and changed weekly. The mice were kept under a 12:12 h light-dark cycle in an environment with a temperature ranging from 21 to $25^{\circ} \mathrm{C}$, a relative humidity of $30-70 \%$, and 10 air shifts $/ \mathrm{h}$. The mice were allowed free access to a pelleted rodent diet (MF, Oriental Yeast Co., Ltd., Tokyo, Japan) and reverse osmosis water with $2 \mathrm{ppm}$ sodium hypochlorite added. These sentinels were housed in 30 animal rooms for 6 months.

To monitor the presence of MNV in our mouse colonies, the sentinel mice were exposed once a month to soiled bedding obtained from about $20 \%$ of the total mouse cages. During the study period, the sentinel mice in all the rooms were found to be devoid of antibodies to a panel of mouse viruses (mouse hepatitis virus, Sendai virus, ectromelia virus, and lymphocytic choriomeningitis virus) and devoid of Mycoplasma pulmonis, Citrobacter rodentium, Clostridium piliforme, Corynebacterium kutscheri, and Salmonella spp. All the animal procedures were approved by the Animal Care and Use Committee of the Osaka University Faculty of Medicine.

\section{$R T-P C R$}

Fresh feces were collected from the mice described above. The collected feces were stored at $-80^{\circ} \mathrm{C}$ until use. RNA was extracted from approximately $30 \mathrm{mg}$ of feces using an RNA extraction kit (RNeasy Mini Kit, Qiagen, Tokyo, Japan). Fecal samples were placed in a homogenate tube, and zirconia beads and $600 \mu$ l of Buffer RLT included in the RNeasy Mini Kit were added. The tube was then capped, and the sample was homogenized for 5 min (30 cycles) using a TissueLyser from Qiagen (Retsch GmbH, Haan, Germany). Following homogenization, samples were centrifuged for $3 \mathrm{~min}$ at 
Table 1. Sequences of MNV-specific primers targeting the MNV RNA polymerase gene

\begin{tabular}{lllc}
\hline \multicolumn{1}{c}{ Name } & \multicolumn{1}{c}{ Sequence (5'-3') } & Location* \\
\hline \multicolumn{2}{c}{ Newly designed primers } & & \\
1st PCR & rpF1 & GAC ATC ATG GTG CGC CTY TCC & $4,323-4,343$ \\
& rpR1 & CTC ATT CAC AAA GAC TGC TG & $5,041-5,060$ \\
2nd (nested) PCR & rpF2 & TGA GGT GGT TTC GAC CAA CC & $4,577-4,596$ \\
& rpR2 & ACC ATT TCT ATC CCA CTA TGG & $4,946-4,966$ \\
\hline Primes reported by Goto et al. & 1st Forward & GAC ATC ATG GTG CGC CT & $4,323-4,339$ \\
1st PCR & 1st Reverse & CTC ATT CAC AAA GAC TGC TG & $5,041-5,060$ \\
\multirow{2}{*}{ 2nd (nested) PCR } & 2nd (nested) F & TCY TTC TAT GGT GAT GAY GA & $4,560-4,579$ \\
& 2nd (nested) R & TCT CAG CAT CCA TTG TTC CA & $5,006-5,025$ \\
\hline
\end{tabular}

*Based on the nucleotide position of the MNV-1 strain complete genome (accession no. AY228235). The new forward primer ( $\mathrm{rpF} 1)$ is extended by 4 nucleotides to the 5 ' side compared with that reported by Goto et al. [2], whereas the reverse primer (rpR1) is identical to that of Goto et al. [2]. The forward primer (rpF2) and reverse primer (rpR2) for the 2nd PCR amplified a 390 bp product.

$20,000 \times g$, and supernatants were transferred to another tube. Subsequently, RNA was extracted according to the manufacturer's instructions and stored at $-80^{\circ} \mathrm{C}$ until use. The RNA samples used for the MNV-positive control were extracted from feces of animals tested by the ICLAS Monitoring Center (Kawasaki, Japan) and found to be positive for MNV by the method reported by Goto et al. [2], with sequences that were more than $90 \%$ similar to the RNA polymerase gene of MNV-1. DNA derived from B. vulgatus (ATCC 8482 D-5) was obtained from the American Type Culture Collection.

PCR primers directed against the MNV RNA polymerase gene used in this study are shown in Table 1. A Reverse Transcriptase M-MLV kit (RNase H free, Takara Bio Inc., Otsu, Shiga, Japan) was used to produce cDNA. Briefly, $12 \mu 1$ extracted RNA, $1 \mu 1$ reverse transcriptase, $1 \mu$ l Oligo dT primer (Novagen, Madison, WI, USA), $2 \mu 1$ of $10 \mathrm{mM}$ dNTPs, and $4 \mu 1$ of the buffer included in the kit were mixed in a tube, and cDNA was generated by heating to $42^{\circ} \mathrm{C}$ for $30 \mathrm{~min}$ and then to $99^{\circ} \mathrm{C}$ for $5 \mathrm{~min}$. The cDNA was immediately cooled on ice and was stored at $-80^{\circ} \mathrm{C}$ until use. PCR kits (PuReTaq Ready-To-Go PCR Beads, GE Healthcare, Buckinghamshire, UK) were used to detect MNV. The first PCR was performed using $18 \mu 1$ DEPC-treated distilled water, 2 $\mu 1$ cDNA, and $2.5 \mu 1$ each of the forward and reverse primers adjusted to a final concentration of $0.25 \mu \mathrm{M}$. The PCR mixture was incubated in a thermal cycler (iCycler, Bio-Rad, Hercules, CA, USA). The reaction conditions were the same as those reported by Goto et al. [2] (Table 2). The second PCR was performed with
Table 2. Reaction conditions used for nested RT-PCR amplification using a set of new primers

\begin{tabular}{lccc}
\hline Step & $\mathrm{n}$ & Temperature & Duration \\
\hline 1 Denaturation & 1 & $94^{\circ} \mathrm{C}$ & $4 \mathrm{~min}$ \\
\hline 2 Cycle & 25 & & \\
Denaturation & & $94^{\circ} \mathrm{C}$ & $1 \mathrm{~min}$ \\
Annealing & & $55^{\circ} \mathrm{C}$ & $1 \mathrm{~min}$ \\
Elongation & & $72^{\circ} \mathrm{C}$ & $1 \mathrm{~min}$ \\
\hline 3 Primer & 1 & & \\
Elongation & & $72^{\circ} \mathrm{C}$ & $5 \mathrm{~min}$ \\
\hline
\end{tabular}

These conditions are the same to those reported by Goto et al. [2] and were used for first/second PCR using a set of new primers.

$2 \mu 1$ of the first PCR product and $23 \mu 1$ of the reaction mixture containing the nested primer set (Table 1), again under the reaction conditions described by Goto et al. [2]. PCR products were subjected to electrophoresis using a $1.5 \%$ agarose gel at $100 \mathrm{~V}$ for $30 \mathrm{~min}$.

\section{PCR for detection of $B$. vulgatus}

PCR detection of $B$. vulgatus was carried out by the method reported by Wang et al. [20]. PCR primers directed against $B$. vulgatus $16 S$ rRNA were as follows: 5'-GCATCATGAGTCCGCATGTTC-3', forward, and 5'-TCCATACCCGACTTTATTCCTT-3', reverse. DNA derived from $B$. vulgatus (ATCC 8482 D-5) was used as a control.

\section{Removing DNA from RNA extracts}

To avoid nonspecific PCR reactions, DNA was re- 
moved from RNA extracts using a TURBO DNA-free Kit (Ambion Inc., Austin, TX, USA). The degradation procedure was carried out according to the manufacturer's instructions, and the test samples were stored at $-80^{\circ} \mathrm{C}$ until use.

\section{Nucleotide sequence analysis}

PCR products amplified with MNV or B. vulgatus were purified using a QIAquick PCR Purification Kit (Qiagen) and were used as the template for cycle sequencing. Cycle sequencing reactions were performed by using a BigDye Terminator ver. 1.1 Cycle Sequencing Kit (Applied Biosystems, Life Technologies Japan, Tokyo, Japan). Sequencing of the PCR products was performed with the same primers as used for PCR amplification. Sequencing reactions were then subjected to nucleotide sequencing using a 3100 Genetic Analyzer sequencer (Applied Biosystems, Life Technologies Japan). The sequences were then aligned and compared with the nucleotide sequence data of various Norovirus and Sapovirus members registered in the GenBank database (http://www.ncbi.nlm.nih.gov/Genbank/GenbankOverview.html), including human Norwalk virus (M87661), Sapporo virus (U65427), and Hawaii calicivirus (U07661); bovine Jena bovine enteric calicivirus (AJ011099); and murine MNV-1 (AY228235), MNV-2 (DQ223041), MNV-3 (DQ223042), MNV-4 (DQ223043), MNV-5 (EF650480), MNV-6 (EF650481), and murine norovirus (AB469416) using the ClustalW software.

\section{Analysis software}

BioEdit version 7.0.5.3 (http://www.mbio.ncsu.edu/ BioEdit/bioedit.html) was used for nucleotide sequence analysis, TreeView version 1.6.6 was used to generate a phylogenetic tree, and ClustalW version 1.83 was used in the phylogenetic analysis.

\section{Antibody testing}

Twenty-four sentinel mice housed in $3 \mathrm{MNV}$-contaminated animal rooms of our animal facilities were euthanized with $\mathrm{CO}_{2}$ and bled out by cardiac puncture to obtain serum. Feces were also collected from these mice to perform nested RT-PCR for MNV. The results were compared with those of serological tests for MNV, which were carried out by CRJ. In the laboratory, primary tests were conducted using a multiplexed fluorescent immunoassay (MFI), and positives were determined by indirect fluorescence antibody (IFA) assays.

\section{Statistical analysis}

The CORREL function in Excel 2003 was used to compute correlation coefficients between data sets from MNV antibodies and PCR test results.

\section{Results}

A previously reported $M N V$-specific primer set detected both $M N V$-specific and B. vulgatus-derived signals

We performed nested RT-PCR on mouse feces using a primer set targeting the RNA polymerase gene that had been previously reported for MNV detection [2]. However, this MNV-specific primer set was found to detect not only MNV-specific signals but also B. vulgatus-derived signals as follows. A majority of the samples (ON1-18) already diagnosed as MNV-positive by the ICLAS Monitoring Center using RT-PCR yielded a strong signal with a size of approximately $466 \mathrm{bp}$ (Fig. 1A, lanes 1-18). Sequencing of the 466-bp PCR products excised from the agarose gel confirmed that they were all derived from the RNA polymerase gene of MNV. Hereafter, we used these RNA samples as MNV-positive controls for RT-PCR.

Phylogenetic analysis indicated that these sequences fell into the same cluster as those obtained from known MNVs (Fig. 1B). Unexpectedly, however, nucleotide sequences obtained from 2 samples (ON19 and ON20) yielded a faint band of approximately 466 bp (Fig. 1A, lanes 19 and 20); these sequences fell into a completely different cluster, indicating that they were not derived from the norovirus RNA polymerase gene (Fig. 1B, ON19 and ON20). Additionally, these samples were obtained from MNV-seronegative animals (data not shown). DDBJ BLAST software analysis indicated that the sequences obtained from the faint band were $98.6 \%$ (ON20) and 99.3\% (ON19) identical, respectively, to those of the gene encoding the exophosphatase gene of B. vulgatus (GenBank accession no. CP000139; Fig. 2), suggesting that these $\mathrm{MNV}$-specific primers we had used were cross-reactive with the $B$. vulgatus genome.

We therefor examined whether the primers we used reacted with the $B$. vulgatus genome. Our results suggested that the 3 '-end of the MNV-specific primers was partly homologous with the $B$. vulgatus nucleotide sequence. Specifically, a 7-bp-long region (5'-ATGAYGA-3') of the reverse primer and a 14-bp-long region (5'-CATCCATTGTTCCA-3') of the forward primer used for the second PCR were found to be complementary to 
(A)

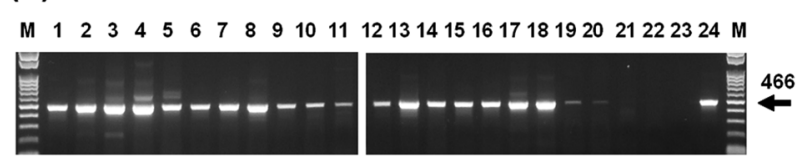

(B)

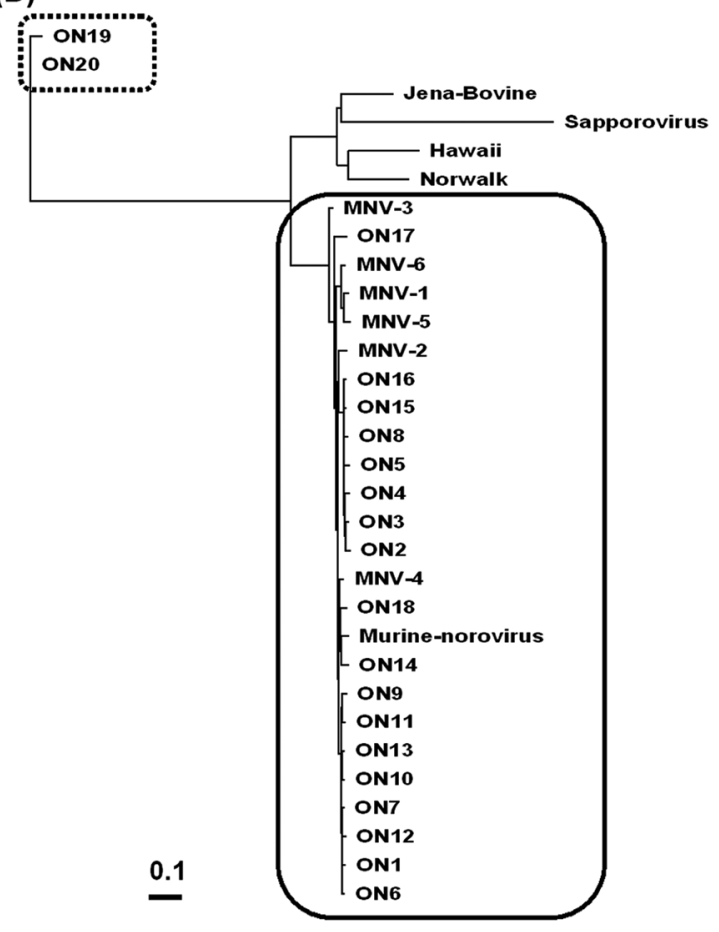

Fig. 1. MNV detection using RT-PCR products amplified with a set of primers originally described by Goto et al. RNA extracts derived from sentinel mice housed in our animal facilities were used. (A) Gel electrophoresis of PCR products. Lanes: M, 100-bp ladder; 1-22, RNA extracts derived from sentinel mice (ON1-22); 23, water; 24, MNV-positive control. The arrow indicates the PCR product size. (B) Phylogenetic analysis of the nucleotide sequences obtained from the PCR products indicated in the upper panel and from the reference samples (MNV and related viruses). The scale bar indicates an evolutionary distance of 0.1 between sequences.

sequences found in the B. vulgatus genome; these sequences are underlined in Fig. 2.

\section{B. vulgatus-derived DNA was frequently found in RNA extracted from feces}

We next examined whether $B$. vulgatus-derived DNA existed in feces using a specific set of primers for $B$. vulgatus $16 \mathrm{~S}$ rRNA. PCR was carried out using $6 \mathrm{RNA}$ extract samples that were derived from MNV-seropositive (Fig. 1, lanes 8 and 11) and MNV-seronegative (Fig. 1, lanes 19-22) mice. In 5 out of 6 RNA extract samples, we observed a single prominent band with a size of approximately 287 bp (Fig. 3A, upper panel, lanes 2-6). This band was also observed in $B$. vulgatus-derived DNA (lane 7). Only 1 sample out of 6 was negative for this band (lane 1). DNA degradation of RNA extracts by DNase treatment before the RT reaction resulted in a complete loss of this 287-bp band in the feces (Fig. 3A, lower panel). These data supported the hypothesis that B. vulgatus DNA that cross-reacted with this MNVspecific primer set was in fact sometimes present in feces, obscuring interpretation of RT-PCR data for MNV detection.

We then tested whether the MNV-specific primer set reported by Goto et al. [2] could amplify a signal from samples containing $B$. vulgatus-derived DNA using RTPCR. As shown in Fig. 3B, these primers yielded both a thin band of approximately $466 \mathrm{bp}$ (upper panel, lanes 3 and 4) and a fuzzy band of a smaller size from purified B. vulgatus DNA (upper panel, lane 7). Corresponding to the data shown in Fig. 1, these primers gave a prominent band of 466 bp in 2 fecal samples (ON8 and ON11) obtained from serologically MNV-positive animals (Fig. 3B, upper panel, lanes 1 and 2). They also gave a thin band of a similar size from 2 fecal samples (ON19 and ON20) obtained from serologically MNV-negative animals (upper panel, lanes 3 and 4 ) and from B. vulgatus DNA (upper panel, lanes 7). However, the samples did not amplify any signal from 2 other MNV-seronegative animals (ON21 and ON22; upper panel, lanes 5 and 6). Moreover, when DNA was removed from these RNA extracts using DNase treatment, the weak band obtained from $2 \mathrm{MNV}$-seronegative mice (ON19 and ON20) and that from B. vulgatus DNA were completely abolished (Fig. 3B; lower panel, lanes 3, 4, and 7), whereas the strong band obtained from the $2 \mathrm{MNV}$-seropositive mice was unaffected (ON8 and ON11, lower panel, lanes 1 and 2). These results demonstrate that the MNV-specific primer set reported by Goto et al. [2] cross-reacted with $B$. vulgatus DNA, giving a false-positive 466-bp band in some of the MNV-negative mice. The identity of the small fuzzy band obtained from purified $B . v u l$ gatus DNA with the primers reported by Goto et al. [2] remains unclear.

Development of new primers targeting the MNV RNA polymerase gene

In order to avoid having to treat fecal RNA extracts with DNase, we next sought to develop a new set of 


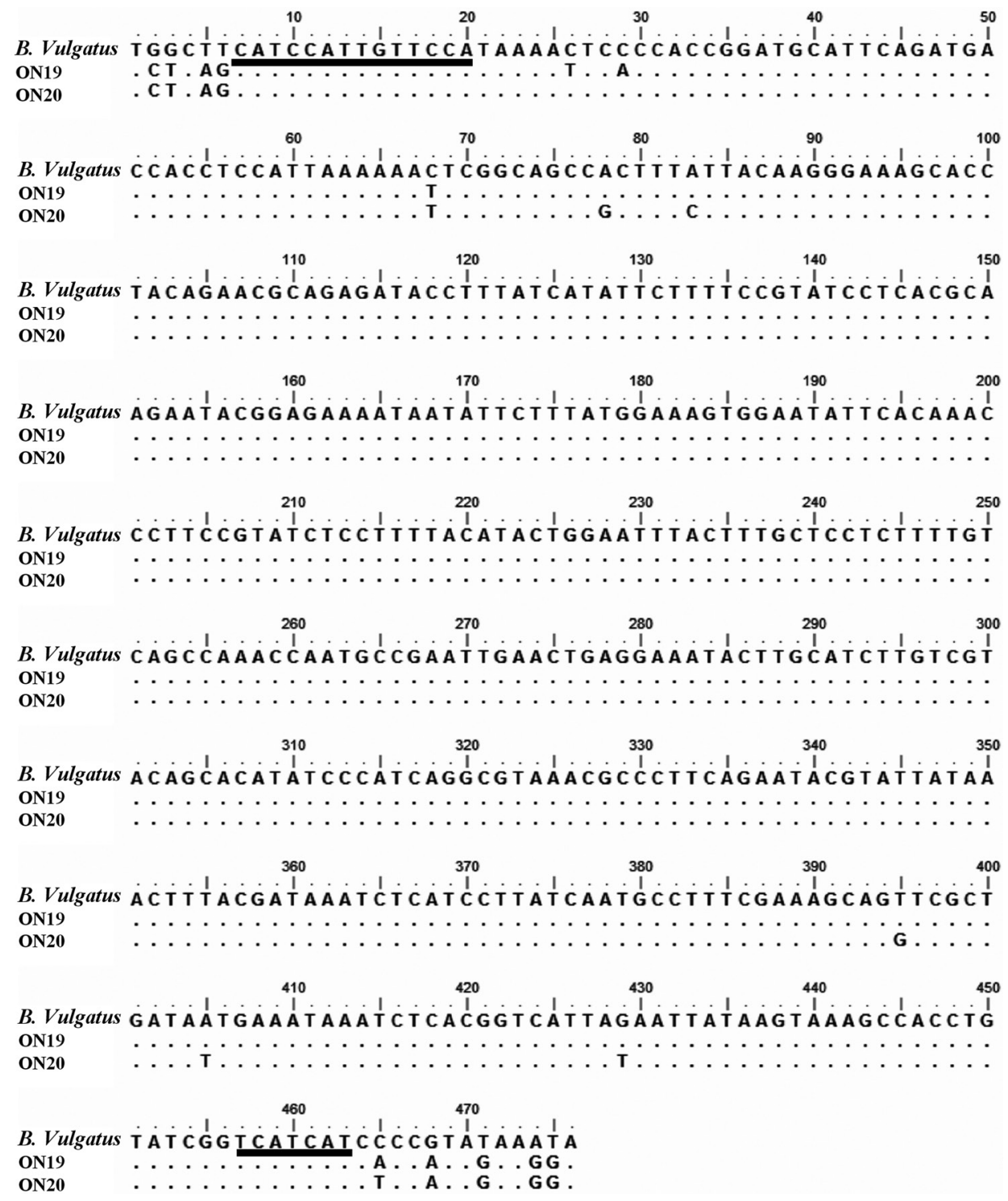

Fig. 2. Nucleotide sequence alignment of a partial B. vulgatus sequence encoding the exophosphatase gene and sequences derived from the PCR products for MNV (ON19 and ON20). A dot represents the same nucleotide as that found in B. vulgatus. Underlining indicates the same nucleotides as those of the second forward or reverse primers described by Goto et al.

highly specific MNV primers that would not cross-react with the B. vulgatus genome. The new second PCR primers, shown in Table 1, were directed against a less crossreactive region in the MNV RNA polymerase gene and amplified a 390-bp product. The first PCR forward primer was also modified by adding 4 nucleotides $\left(5^{\prime}-\mathrm{Y}\right.$ TCC-3') to the 3'-end in order to avoid cross-reaction. The PCR conditions were the same as those reported by Goto et al. [2].

When these primers were used for nested RT-PCR, a 390-bp product was detected from untreated RNA ex- tracts obtained from the feces of MNV-seropositive animals (Fig. 4, ON8 and ON11, lanes 1 and 2), while no signal was detected from the RNA samples obtained from the feces of MNV-seronegative, B. vulgatus-positive animals (ON19-22, lanes 3-6). Moreover, no 390-bp product was detected from purified, DNase-untreated $B$. vulgatus DNA (lane 7). These results indicated that our new primers specifically amplified only the MNV genome. Supporting this notion, nucleotide sequencing of the 390-bp band of $18 \mathrm{MNV}$-positive animals revealed $89.3-92.2 \%$ identity to the sequence of the MNV-1 RNA 
(A)

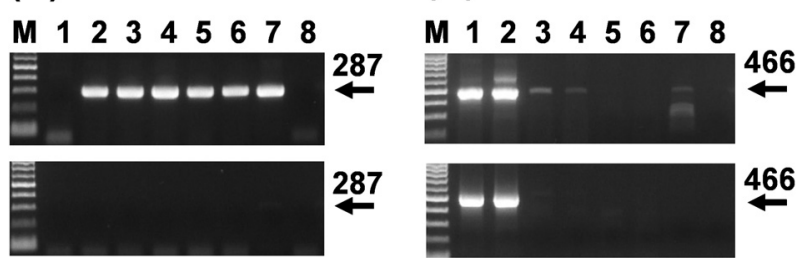

Fig. 3. PCR or RT-PCR products obtained from RNA extracts of untreated fecal matter or RNA extracts subjected to DNase treatment for DNA removal and amplified with $B$. vulgatus16S rRNA primers or MNV-specific primers described by Goto et al. Arrows indicate the PCR product size. (A) PCR products amplified with $B$. vulgatus-16S $r R N A$ primers. Upper panel, untreated RNA extracts; lower panel, RNA extracts subjected to DNase treatment for DNA removal before PCR. (B) RT-PCR products amplified with MNVspecific primers. Upper panel, untreated RNA extracts; lower panel, RNA extracts subjected to DNase treatment for DNA removal before reverse transcription. (A, B) Lanes: M, 100-bp ladder; 1 and 2, MNV-seropositive mice (ON8 and ON11); 3-6, MNV-seronegative mice (ON1922); 7, B. vulgatus-derived DNA; 8, water.

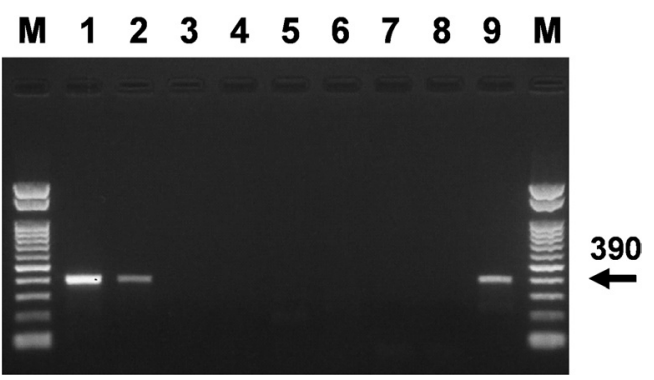

Fig. 4. Gel electrophoresis of RT-PCR products amplified with a new set of MNV-specific primers using DNase-untreated RNA extracts. The arrow indicates the PCR product size. Lanes: M, 100-bp ladder; 1 and 2, fecal RNA extracts from MNV-seropositive animals (ON8 and ON11); 3-6, RNA extracts from MNV-seronegative animals (ON19-22); 7, B. vulgatusderived DNase-untreated DNA; 8, water; 9, MNV-positive control.

polymerase gene for the nucleotide sequence and 97.3$99.1 \%$ identity for the amino acid sequence (Table 3 ). In addition, when the RT-PCR data based on the new primers were compared with the data obtained by an MNV antibody test using 24 different sentinel animals housed in $3 \mathrm{MNV}$-contaminated rooms of our animal facilities, 4 PCR-positive animals were antibody positive, while 19 out of 20 PCR-negative animals were MNV seronegative and 1 was seropositive (Table 4), showing a strong posi-
Table 3. Extent of identity of the 390-bp band to the MNV-1 RNA polymerase gene

\begin{tabular}{ccc}
\hline & \multicolumn{2}{c}{ Sequence identity (\%) } \\
\cline { 2 - 3 } Sample no & Amino acid ${ }^{\text {a) }}$ & Nucleotide $^{\mathrm{b})}$ \\
\hline ON1 & 98.2 & 89.9 \\
ON2 & 99.1 & 90.5 \\
ON3 & 99.1 & 89.3 \\
ON4 & 99.1 & 89.6 \\
ON5 & 98.2 & 90.2 \\
ON6 & 98.2 & 91.4 \\
ON7 & 98.2 & 90.2 \\
ON8 & 97.3 & 90.8 \\
ON9 & 99.1 & 91.4 \\
ON10 & 98.2 & 91.1 \\
ON11 & 99.1 & 90.2 \\
ON12 & 98.2 & 89.9 \\
ON13 & 98.2 & 92.2 \\
ON14 & 99.1 & 89.9 \\
ON15 & 98.2 & 90.5 \\
ON16 & 99.1 & 90.2 \\
ON17 & 99.1 & 90.8 \\
ON18 & 97.3 & 90.8 \\
\hline
\end{tabular}

a) 115 amino acids. b) 349 bp nucleotides. The percentage of the sequence identity between a part of the RNA polymerase gene of MNV-1 and samples (Fig. 1, ON1-ON18) was calculated using the BioEdit software as described in Materials and Methods. The sequences were obtained from 390-bp PCR products amplified with our new primers using RNA extract samples.

Table 4. Correlation of the results between RT-PCR and antibody tests for MNV of sentinel mice

\begin{tabular}{lcc}
\hline \multirow{2}{*}{ RT-PCR $^{\text {b) }}$} & \multicolumn{2}{c}{ Antibody $^{\text {a) }}$} \\
\cline { 2 - 3 } & Positive & Negative \\
\hline Positive & 4 & 0 \\
Negative & 1 & 19 \\
\hline
\end{tabular}

The results of the antibody tests and RT-PCR were obtained from 24 sentinel mice housed in $3 \mathrm{MNV}$-contaminated rooms of our animal facilities. ${ }^{\text {a) Anti- }}$ body test was performed by CRJ labo-

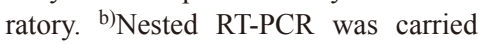
out using a newly designed set of PCR primers. Correlation coefficient $=0.872$.

tive correlation between the results obtained from the two tests (correlation coefficient $=0.872$ ).

\section{Discussion}

Here, we report that an RT-PCR-based MNV detection test using a previously reported primer set [2] detected 
not only an MNV-specific signal, but also a signal derived from a commensal bacterium, B. vulgatus; the nucleotide sequence of the latter signal showed at least 98\% identity with that of the exophosphatase gene of $B$. vulgatus (Fig. 1, ON19 and ON20). These faint bands disappeared after removing contaminated DNA from fecal RNA extracts (Fig. 3B). While degradation of DNA from RNA extract may help reduce false-positive results, this process may prolong the period of sample preparation. Moreover, because this bacterium is commonly found in the SPF laboratory mouse intestine [13], the primers reported by Goto et al. [2] are likely to confound the interpretation of MNV tests using fecal samples, unless the DNA was first removed. Thus, we designed a new primer set that obviates the need for DNA removal before RT-PCR analysis.

Detecting MNV infection has so far depended mainly on antibodies and RT-PCR assays. The latter method has been used frequently because it allows not only early detection of MNV RNA during MNV infection but also identification of the virus origin through nucleotide sequencing of the PCR products [22]. Primers used to detect MNV have been directed against highly conserved nucleotide MNV sequences, such as those representing the $R N A$ polymerase gene $[2,6]$ and ORF $1 / O R F 2$ of the MNV genome [10, 14]. On the other hand, the primer set reported by Goto et al. [2] was thought to be selectively directed against the MNV RNA polymerase gene. Here, we found that this primer set cross-reacted with the B. vulgatus genome; the 3'-end of the MNV-specific primers in the second PCR reaction was homologous with the $B$. vulgatus nucleotide sequence, and this homology appeared to make them cross-reactive with $B$. vulgatus DNA, incidentally yielding a signal very similar in size to an MNV-specific PCR product. Although this cross-reactivity may be circumvented by re-evaluating the annealing temperature conditions or altering the amount of primers used, given that $B$. vulgatus is a commensal bacterium [13], the bacterial DNA will inevitably be present in fecal samples at high frequency, and thus, these primers cannot be relied upon unless the DNA is completely removed from the fecal samples. While real-time RT-PCR assays detecting MNV RNA have been reported recently $[1,4,11]$, rigorous examination will be required to verify the assay specificity.

We thus designed new MNV-specific primers that have no cross-reactivity with $B$. vulgatus. First, we performed nested PCR with a newly designed second PCR primer set and the first primer set reported by Goto et al. using $B$. vulgatus DNA. Although the nonspecific thin band disappeared, the unidentified small fuzzy band did not disappear (data not shown). Finally, we modified a forward primer for the first PCR (rpF1) reported by Goto et al. by adding 4 nucleotides to improve the assay specificity (Table 1). When we used these newly designed primers, we found a 390-bp signal in RNA samples from the feces of MNV-seropositive animals, but not from the feces of MNV-seronegative, B. vulgatuspositive animals. We also did not detect a signal from $B$. vulgatus DNA. Nucleotide sequencing of the 390-bp band verified that it was indeed derived from MNV (Table 3). We then compared these results with those obtained by an MNV antibody test using 24 different sentinel animals and found a strong correlation between these 2 tests (Table 4), further strengthening the notion that the newly designed set of primers could be used to detect an MNV-specific signal. It is of note, however, that 1 out of 20 PCR-negative samples was antibody positive; one possible explanation is that the MNV genome passed only temporarily into the feces despite chronic infection of this animal. Hsu et al. also reported that MNV genomes could be detected from feces of some but not all MNV-seropositive mice [5].

Collectively, our study indicates a potential pitfall in detecting MNV infection by RT-PCR using fecal samples. The new primer set designed in the present study should be useful for reliable RT-PCR-based MNV detection in fecal samples.

\section{References}

1. Bae, J. and Schwab, K.J. 2008. Evaluation of murine norovirus, feline calicivirus, poliovirus, and MS2 as surrogates for human norovirus in a model of viral persistence in surface water and groundwater. Appl. Environ. Microbiol. 74: 477-484. [Medline] [CrossRef]

2. Goto, K., Hayashimoto, N., Yasuda, M., Ishida, T., Kameda, S., Takakura, A., and Itoh, T. 2009. Molecular detection of murine norovirus from experimentally and spontaneously infected mice. Exp. Anim. 58: 135-140. [Medline] [CrossRef]

3. Henderson, K.S. 2008. Murine norovirus, a recently discovered and highly prevalent viral agent of mice. Lab Anim. (NY) 37: 314-320. [Medline] [CrossRef]

4. Hewitt, J., Rivera-Aban, M., and Greening, G.E. 2009. Evaluation of murine norovirus as a surrogate for human norovirus and hepatitis A virus in heat inactivation studies. J. Appl. Microbiol. 107: 65-71. [Medline] [CrossRef]

5. Hsu, C.C., Riley, L.K., Wills, H.M., and Livingston, R.S. 2006. Persistent infection with and serologic cross-reactivity 
of three novel murine noroviruses. Comp. Med. 56: 247-251. [Medline]

6. Hsu, C.C., Wobus, C.E., Steffen, E.K., Riley, L.K., and Livingston, R.S. 2005. Development of a microsphere-based serologic multiplexed fluorescent immunoassay and a reverse transcriptase PCR assay to detect murine norovirus 1 infection in mice. Clin. Diagn. Lab. Immunol. 12: 1145-1151. [Medline]

7. Karst, S.M., Wobus, C.E., Lay, M., Davidson, J., and Virgin, H.W. 2003. STAT1-dependent innate immunity to a Norwalk-like virus. Science 299: 1575-1578. [Medline] [CrossRef]

8. Kelmenson, J.A., Pomerleau, D.P., Griffey, S., Zhang, W., Karolak, M.J., and Fahey, J.R. 2009. Kinetics of transmission, infectivity, and genome stability of two novel mouse norovirus isolates in breeding mice. Comp. Med. 59: 27-36. [Medline]

9. Kitagawa, Y., Tohya, Y., Ike, F., Kajita, A., Park, S.J., Ishii, Y., Kyuwa, S., and Yoshikawa, Y. 2010. Indirect ELISA and indirect immunofluorescent antibody assay for detecting the antibody against murine norovirus S7 in mice. Exp. Anim. 59: 47-55. [Medline] [CrossRef]

10. Kitajima, M., Oka, T., Tohya, Y., Katayama, H., Takeda, N., and Katayama, K. 2009. Development of a broadly reactive nested reverse transcription-PCR assay to detect murine noroviruses, and investigation of the prevalence of murine noroviruses in laboratory mice in Japan. Microbiol. Immunol. 53: 531-534. [Medline] [CrossRef]

11. Kitajima, M., Oka, T., Takagi, H., Tohya, Y., Katayama, H., Takeda, N., and Katayama, K. 2010. Development and application of a broadly reactive real-time reverse transcription-PCR assay for detection of murine noroviruses. J. Virol. Methods 169: 269-273. [Medline] [CrossRef]

12. Lencioni, K.C., Seamons, A., Treuting, P.M., Maggio-Price, L., and Brabb, T. 2008. Murine norovirus: an intercurrent variable in a mouse model of bacteria-induced inflammatory bowel disease. Comp. Med. 58: 522-533. [Medline]

13. Momose, Y., Park, S.H., Miyamoto, Y., and Itoh, K. 2011. Design of species-specific oligonucleotide probes for the detection of Bacteroides and Parabacteroides by fluorescence in situ hybridization and their application to the analysis of mouse caecal Bacteroides-Parabacteroides microbiota. J. Appl. Microbiol. 111: 176-184. [Medline] [CrossRef]

14. Muller, B., Klemm, U., Marques, A.M., and Schreier, E. 2007. Genetic diversity and recombination of murine noroviruses in immunocompromised mice. Arch. Virol. 152:
1709-1719. [Medline] [CrossRef]

15. Mumphrey, S.M., Changotra, H., Moore, T.N., HeimannNuchols, E.R., Wobus, C.E., Reilly, M.J., Moghadamfalahi, M., Shukla, D., and Karst, S.M. 2007. Murine norovirus 1 infection is associated with histopathological changes in immunocompetent hosts, but clinical disease is prevented by STAT1-dependent interferon responses. J. Virol. 81: 32513263. [Medline] [CrossRef]

16. Paik, J., Fierce, Y., Drivdahl, R., Treuting, P.M., Seamons, A., Brabb, T., and Maggio-Price, L. 2010. Effects of murine norovirus infection on a mouse model of diet-induced obesity and insulin resistance. Comp. Med. 60: 189-195. [Medline]

17. Perdue, K.A., Green, K.Y., Copeland, M., Barron, E., Mandel, M., Faucette, L.J., Williams, E.M., Sosnovtsev, S.V., Elkins, W.R., and Ward, J.M. 2007. Naturally occurring murine norovirus infection in a large research institution. J. Am. Assoc. Lab. Anim. Sci. 46: 39-46. [Medline]

18. Pritchett-Corning, K.R., Cosentino, J., and Clifford, C.B. 2009. Contemporary prevalence of infectious agents in laboratory mice and rats. Lab. Anim. 43: 165-173. [Medline] [CrossRef]

19. Thackray, L.B., Wobus, C.E., Chachu, K.A., Liu, B., Alegre, E.R., Henderson, K.S., Kelley, S.T., and Virgin, H.W. 2007. Murine noroviruses comprising a single genogroup exhibit biological diversity despite limited sequence divergence. $J$. Virol. 81: 10460-10473. [Medline] [CrossRef]

20. Wang, R.F., Cao, W.W., and Cerniglia, C.E. 1996. PCR detection and quantitation of predominant anaerobic bacteria in human and animal fecal samples. Appl. Environ. Microbiol. 62: 1242-1247. [Medline]

21. Wobus, C.E., Karst, S.M., Thackray, L.B., Cang, K.O., Sosnovtsev, S.V., Belliot, G., Krug, A., Mackenzie, J.M., Green, K.Y., and Virgin, H.W. 2004. Replication of norovirus in cell culture reveals a tropism for dendritic cells and macrophages. PLoS Biol. 2: e432. [Medline] [CrossRef]

22. Yamada, Y.K., Yabe, M., Kyuwa, S., Nakamura, N., Takimoto, K., and Urano, T. 2001. Differentiation of mouse hepatitis viruses in animal facilities in Japan by use of nucleotide analysis of the nucleocapsid gene. Comp. Med. 51:319-325. [Medline]

23. Yeom, S.C., Yu, S.A., Choi, E.Y., Lee, B.C., and Lee, W.J. 2009. Prevalence of Helicobacter hepaticus, murine norovirus, and Pneumocystis carinii and eradication efficacy of cross-fostering in genetically engineered mice. Exp. Anim. 58: 497-504. [Medline] [CrossRef] 